\title{
Representação fonológica dos róticos do Português Brasileiro: uma abordagem à base de exemplares
}

\author{
Iiris Rennicke*
}

\begin{abstract}
Este artigo examina a variação dos róticos, ou sons de $r$, em um corpus de português brasileiro coletado em Lavras - MG. Uma análise acústica revelou 21 variantes que envolvem vibrantes, tepes, fricativas, aproximantes e aproximantes aspiradas, podendo ser alveolares, palatais, retroflexas/ arqueadas, uvulares e glotais. A gradiência fonética observada em todos os contextos fonológicos tem como motivação a lenição (redução), que pode diminuir a magnitude do gesto articulatório ou realinhar os gestos temporalmente. De acordo com a Fonologia de Exemplares, a lenição ocorre pela automatização de padrões neuromotores. Utilizamos a gradiência observada nos dados, completada com informações sobre mudanças históricas, para argumentar que a classe dos róticos se justifica não como classe natural, mas como uma rede de relações de parentesco específicas de uma língua. As ligações entre variantes róticas, nem sempre presentes na fala de indivíduos, podem ser resgatadas e estabelecidas neste tipo de modelo, já que a variação sincrônica impossibilita a definição de traços comuns a todos os róticos. A rede de róticos é específica de cada língua porque é formada em interação com outros elementos do sistema fonológico. A sobreposição de variantes anteriores e posteriores em coda silábica indica que o contraste entre R-forte e r-fraco pode ser caracterizado como um "quase-contraste".
\end{abstract}

Palavras-chave: Róticos. Fonologia de Exemplares. Lenição. Classe fonológica.

\section{Introdução}

Este artigo tem por objetivo discutir a representação fonológica dos róticos, ou sons de $r$, no português brasileiro (doravante PB), através de um corpus de fala coletado em Lavras - MG, com falantes das mesorregiões do Campo das Vertentes, Sul e Sudoeste de Minas e Oeste de Minas. A variação na realização dos róticos, especialmente em coda silábica, caracteriza variedades geográficas e sociais do PB. Por este motivo, os róticos têm sido o foco de estudos variacionistas (CALLOU; MORAES; LEITE, 1996; OLIVEIRA, 1983, 1997; LEITE, 2004,

1 Universidade Federal de Minas Gerais, UFMG. PhD, Faculdade de Letras. 
2010; HUBACK, 2006) e descrições dialetológicas do PB (ZÁGARI, 1998; NOLL, 2008, p. 71). O contraste incompleto entre o r-fraco (caro) e o R-forte (carro), que se manifesta somente em posição intervocálica, tem inspirado análises da representação fonológica dos róticos (por exemplo, ABAURRE; SANDALO, 2003; MONARETTO, QUEDNAU; HORA, 2005, p. 215-220, sobre o PB; MATEUS; D'ANDRADE, 2000, p. 15-16, sobre o português europeu, doravante PE; e BONET; MASCARÓ, 1997, sobre as línguas ibero-românicas em geral), nas quais é recorrente a ideia de que este contraste incompleto implica uma única forma subjacente.

O propósito deste artigo, porém, é discutir a representação dos róticos a partir de dados fonéticos minuciosos, estabelecendo contínuos gradientes entre variantes róticas. A gradiência observada nos dados entre falantes, entre diversas ocorrências do mesmo contexto fonológico, entre realização e cancelamento do rótico, e entre r-fraco e R-forte, traz novos níveis de complexidade que as análises de natureza formal ou binária não têm levado em consideração. Uma descrição e uma análise detalhadas do caráter variável e gradiente dos róticos são de interesse não só para a área da Linguística, mas também para áreas como a Tecnologia da Fala e a Fonoaudiologia, que dependem de pesquisas meticulosas sobre a variabilidade dos róticos em suas aplicações.

Após esta introdução, o artigo continuará com uma explicação do marco teórico e conceitos adotados na Seção 2. A seguir, na seção 3, serão exploradas as mudanças sonoras sofridas pelos róticos que levaram à distribuição atual. A seção 4 apresentará informações sobre o corpus e os métodos utilizados para a sua observação. Na seção 5, apresentaremos as variantes atestadas no corpus e os contextos em que elas ocorrem. A gradiência evidente nas variantes róticas e as suas implicações para qualquer análise fonológica serão discutidas na seção 6 . À base desta discussão da gradiência, a seção 7 trará uma explicação dos róticos como uma classe de sons emergente que se constrói como uma rede de variantes à base de efeitos de frequência. Finalmente, a seção 8 resumirá os argumentos deste artigo. 


\section{Marco teórico}

\subsection{Modelos de Exemplares}

Adotamos o marco teórico dos Modelos de Exemplares (JOHNSON, 1997, 2007; BYBEE, 2001，2006，2010; PIERREHUMBERT, 2001，2002; CRISTÓFARO-SILVA, 2006), segundo os quais a representação fonológica é individual, acumulativa e probabilística. De acordo com esta teoria, da mesma forma que as experiências repetidas formam e fortalecem as nossas lembranças enquanto experiências menos frequentes são esquecidas, exemplares de experiência linguística formam a base da representação linguística de cada falante. Uma introdução extensa aos Modelos de Exemplares aplicados à fonologia pode ser encontrada em JOHNSON (2007) e RENNICKE (2015, p. 62-75).

Para a maioria dos fonólogos com um enfoque de exemplares, como JOHNSON $(1997,2007)$ e BYBEE $(2001,2006)$, a experiência direta do falante leigo é com palavras inteiras, e não com sons. A cada ocorrência de uma palavra em uma interação linguística, o falante registrará um exemplar dessa palavra, que terá ligações fonéticas, semânticas e morfológicas com outras palavras. Estas ligações podem formar generalizações ou esquemas (schemas, cf. BYBEE 2001, p. 21-28), que servem como base do conhecimento gramatical do falante. Por este motivo, os modelos de exemplares não carecem de generalizações estruturais; a diferença com relação aos modelos formais é que, enquanto estes assumem que formas de superfície têm a sua origem em categorias gramaticais fixadas na infância, os modelos de exemplares assumem que o uso da língua cria e atualiza as generalizações estruturais ao longo da vida do falante.

$\mathrm{Na}$ Fonologia de Exemplares, as categorias fonológicas emergem à base da frequência com que o falante encontra diversas variantes fonéticas em um certo contexto fonológico. As categorias fonológicas têm membros mais centrais (mais frequentes) e mais periféricas (menos frequentes), e essa dinâmica é atualizada constantemente através do uso. A variabilidade registrada tanto no nível lexical quanto nas generalizações que formam categorias fonológicas explica por que os falantes são capazes de perceber e produzir um contínuo de variantes fonéticas para a mesma palavra ou categoria.

Neste artigo, preferimos referir-nos a categorias fonológicas em vez de fonemas devido à diferença fundamental na sua definição: nos modelos fonológicos formais, o fonema é visto como uma única forma subjacente, definida em termos 
de traços distintivos, da qual as formas de superfície são derivadas através de regras. Esta visão de subjacência implica que, na mudança sonora, uma regra é aplicada à forma subjacente para transformá-la na nova forma de superficie. No entanto, este raciocínio também implica que a forma subjacente, mesmo com a mudança sonora, se mantém imutável e poderá voltar a ocorrer na superfície caso a mudança sonora seja impedida. Porém, de acordo com Bybee (2001), uma mudança sonora nunca poderá voltar atrás já que tem um efeito permanente na representação lexical (BYBEE, 2001, p. 59). A variação fonética nos róticos a ser discutida neste artigo que se manifesta tanto no falante quanto entre falantes - é mais bem acomodada em um modelo flexível que permite representações múltiplas.

\subsection{Lenição como fenômeno essencialmente gestual}

Assumimos uma visão essencialmente fonética dos mecanismos de lenição. $\mathrm{O}$ segmento resultante da lenição pode ser articulatoriamente mais complexo do que o segmento original: BAUER (2008) explica que a lenição de, por exemplo, uma oclusiva intervocálica a uma fricativa acontece por diminuição do gesto articulatório, mas o segmento resultante é, do ponto de vista fonético, mais complexo e preciso do que uma oclusiva. Por este motivo, baseamos a nossa definição de lenição no processo articulatório de enfraquecimento de gestos, e não na qualidade do segmento resultante (BAUER, 2008; SEBREGTS, 2014, p. 26-28). Apoiamo-nos em autores como BROWMAN; GOLDSTEIN (1986; 1992), MOWREY; PAGLIUCA (1995) e BYBEE (2001, p. 73-74), que explicam a lenição como articulação não atingida (articulatory undershoot). Os dois fenômenos que levam à articulação não atingida são a "redução gestual”, ou diminuição da magnitude do gesto articulatório (gestural reduction, ou substantive reduction), e a "redução temporal", ou realinhamento de gestos articulatórios (articulatory retiming, ou temporal reduction). Um exemplo do primeiro seria a mudança de uma oclusiva para uma fricativa ou aproximante, em que uma constrição completa se torna uma constrição com fluxo de ar (turbulenta ou periódica). Um exemplo do segundo seria a nasalização de $\mathrm{Vm} \sim \mathrm{Vn} \rightarrow \tilde{\mathrm{V}} \mathrm{m} \sim \tilde{\mathrm{V}} \mathrm{n} \rightarrow \tilde{\mathrm{V}}$ em que o abaixamento da úvula começa a ocorrer antes da constrição da consoante, resultando em uma sobreposição dos gestos vocálico e nasal. A redução gestual e a temporal são causadas pela repetição de palavras e das configurações articulatórias sequenciais e simultâneas que as compõem, o que automatiza padrões neuromotores ao longo do tempo. (BYBEE, 2001, 2006) 


\section{Panorama das mudanças sonoras nos róticos}

Em RENNICKE (2015, p. 40-47) sugerimos que os róticos do português passaram por três principais fases de mudança sonora: 1) lenição de vibrantes ([r]) a tepes ([r]); 2) posteriorização de articulações alveolares a uvulares; e 3) diversificação por meio de lenição em coda silábica na variedade brasileira. Estas três fases serão discutidas nas subseções abaixo.

\subsection{Contraste intervocálico de modo de articulação}

Assume-se que o rótico do latim era uma vibrante alveolar ([r]), que podia ser simples ([r]) ou geminada ([r:]) em posição intervocálica (MATTOSO CAMARA JR, 1953; STURTEVANT, 1920, p. 81-82). Na passagem do latim para o latim vulgar na România ocidental, as consoantes intervocálicas sofreram redução gestual e temporal, resultando em mudanças na duração e/ou no vozeamento: da mesma forma que, por exemplo, $[\mathrm{t}:] \rightarrow[\mathrm{t}],[\mathrm{t}] \rightarrow[\mathrm{d}]$ e $[\mathrm{d}] \rightarrow \varnothing$, o geminado $[\mathrm{r}:] \rightarrow$ [r] e o simples [r] $\rightarrow$ [r]. Os gestos utilizados na produção de [r] e [r] são essencialmente diferentes: [r] requer uma postura fixa do dorso da língua, enquanto [r] é produzido com um movimento balístico envolvendo todo o corpo da língua (CATFORD, 1977, p. 130; BARRY, 1997). Portanto, a mudança $[r] \rightarrow[r]$ não ocorre através de uma simples diminuição de tempo ou do gesto articulatório. Uma possível ligação articulatória seria uma vibrante enfraquecida a uma batida da língua contra os alvéolos, que foi atestada no espanhol peninsular (BLECUA FALGUERAS, 2001). Esta variante enfraquecida de uma batida poderia ser articulatoriamente reinterpretada ao longo do tempo como um tepe pela semelhança perceptiva. Esta primeira fase de mudança sonora fez com que o contraste intervocálico dos róticos do português passasse de um contraste de duração ([r:] : [r]) a um contraste de modo de articulação ([r] : [r]).

\subsection{Posteriorização}

A motivação da posteriorização $[\mathrm{r}] \rightarrow[\mathrm{R}]$ não é clara: não se trata de redução gestual ou temporal. RECASENS (2002) vê esta posteriorização como uma estratégia para diminuir a tensão na ponta da língua mediante um arqueamento 
da lâmina e elevação do dorso da língua em direção ao palato macio. Tratase, portanto, de uma permuta de ponto de articulação sem motivação evidente. SEBREGTS (2014, p. 135) argumenta que o motivo se encontra na aquisição de ponto de articulação "errado" e consequente solidificação por falta de oposições de ponto ou modo no sistema fonológico da língua em questão.

As primeiras referências à vibrante uvular [R] no PE são do final do século XIX (GONÇALVES VIANA, 1973, p. 102; 1903, p.19); não fica claro se esta articulação tinha motivação aquisicional ou se estava sendo adotada por falantes adultos. Meio século depois, $[\mathrm{r}]$ e [R] já estavam sendo substituídas por [x] no português lisboeta (BARBOSA, 1965, p. 187-208). Na virada dos séculos XX e XXI, as fricativas uvulares e velares já são as variantes mais frequentes no PE (RENNICKE; MARTINS, 2013). Assumimos que a posteriorização e fricativização tenham iniciado no Brasil na mesma época que em Portugal (CALLOU; LEITE; MORAES, 2002, p. 544), e em 1977, MATTOSO CAMARA JR. (1977) afirma que o "caráter posterior que hoje predomina no /r/ forte o afasta do caráter anterior do brando, que é uma vibração da ponta da língua" (MATTOSO CAMARA JR., 1977, p. 79). Mais recentemente, autores como CALLOU; LEITE; MORAES (2002) e ABAURRE; SANDALO (2003) destacaram que o R-forte do PB é predominantemente glotal ([h h]). Esta mudança explica-se como debucalização, sendo um tipo de redução gestual em que os gestos articulatórios supraglotais enfraquecem até restar apenas o gesto glotal, que no caso das fricativas é o fluxo de ar na glote (BYBEE; BECKNER, 2015).

\subsection{Diversificação em coda mediante lenição}

A articulação apical ([r] [r]) do rótico em coda silábica ainda é uma realização frequente no PE (MATEUS; RODRIGUES, 2003) e no PB dos estados do Sul e em algumas variedades de São Paulo (CALLOU; MORAES; LEITE, 1996; BARBOSA; ALBANO 2004; SILVA; CLEMENTE; NISHIDA, 2006; NOLL, 2008, p. 71; OUSHIRO; MENDES, 2013). Em outras regiões do Brasil, o rótico em coda sofreu lenição por meio de duas principais trajetórias: posteriorização e fricativização da mesma forma que o R-forte ([в $\chi \curlyvee \times h \mathrm{~h}]$ ); e aproximantização ([. I. ¿ ə] etc.). ${ }^{1}$

1 Utilizamos o termo "aproximante rótica" para referir-nos às aproximantes que se generalizam como "retroflexo" ou "erre caipira" na literatura. A motivação para tal decisão baseia-se na variação acústica, 
A região geográfica onde predomina a primeira trajetória estende do Rio de Janeiro e Centro de Minas Gerais até os estados do Nordeste e Norte; a segunda, por sua vez, envolve o Sul e Oeste de Minas Gerais, São Paulo, Mato Grosso do Sul, e os estados do Sul (ALMEIDA, 2004; CALLOU; MORAIS; LEITE, 1996; ZÁGARI, 1998; NOLL, 2008, p. 71; RIBEIRO, 2011; NOLL, 2012; LIMA, 2013). O local de gravação, Lavras, situa-se na isófona das duas tendências em coda (AGUILERA; SILVA, 2011).

\subsection{Estado atual}

As mudanças sonoras detalhadas na seção 3 levaram à atual distribuição dos róticos no $\mathrm{PB}$, ilustrada na Tabela 1 .

TABELA 1: Distribuição dos róticos no PB

\begin{tabular}{|c|c|c|}
\hline \multirow{4}{*}{ R-forte } & rato & ['hato] \\
\hline & carro & ['kaho] \\
\hline & genro & ['zẽfv] \\
\hline & guelra & ['gewhe] \\
\hline \multirow{2}{*}{$r$-fraco } & prato & ['prato] \\
\hline & caro & ['karo] \\
\hline \multirow{2}{*}{ Coda neutralizada } & porta & ['pohte] ['porte] ['potte] \\
\hline & lugar & [lu'gah] [lu'gar $] \sim[$ lu'ga. $]$ \\
\hline
\end{tabular}

Análises de cunho formalista têm sugerido um tepe / // subjacente (MATEUS; D'ANDRADE, 2000, p. 15-16; MONARETTO; QUEDNAU; HORA, 2005, p. 215-220) ou uma vibrante/r/ subjacente (ABAURRE; SANDALO, 2003; BONET; MASCARÓ, 1997) para todos os róticos. Nestas propostas, a representação fonológica é minimalista, e o conjunto de regras que geram as variantes posicionais é complexo; isto é, a representação abstrata justifica a união de variantes que diferem na forma superficial. Neste artigo, sugeriremos uma organização exemplar dos itens lexicais que possibilita representar o caráter variável e múltiplo dos róticos. Propomos que os róticos (como quaisquer outros segmentos) fazem parte da representação lexical, dispensando um sistema de regras. Assim, de uma palavra

explicada em mais detalhe na seção 5.3. 
como mar, o falante terá armazenado vários exemplares (como $m a[\mathrm{~s}] \sim m a[\mathrm{~h}] \sim m a[$.$] ),$ e a variante fonética utilizada na pluralização ( $m a[\mathrm{r}] e s)$ ou derivação ( $m a[\mathrm{r}]$ ítimo) explica-se como o uso exclusivo do exemplar $m a[r]$. Esta proposta, explicada em mais detalhe na seção 7, adota a visão de uma representação detalhada e complexa, à qual o acesso é direto e não mediado por regras derivacionais.

\section{Corpus e métodos}

O corpus deste artigo foi coletado em Lavras - MG, em 2013, por meio de entrevistas semi-estruturadas. Os participantes eram estudantes na Universidade Federal de Lavras, incluindo 7 mulheres e 7 homens, oriundos de 9 cidades diferentes, com a idade média de 21,3 anos (entre 19 e 24). As gravações foram realizadas com um gravador Zoom H2n Handy Recorder em formato WAV, com a taxa de amostragem $44,1 \mathrm{kHz}$ e tamanho da amostra 16 bits. As variantes dos róticos foram classificadas e listadas utilizando o programa de análise acústica Praat (BOERSMA; WEENINK, 2016) observando no espectrograma a estrutura formântica, indicadores de grau de constrição (corrente de ar periódica ou turbulenta) e vozeamento. A duração das entrevistas variou entre 16 e 32 minutos, gerando 7.045 ocorrências de róticos. Após as entrevistas, os participantes realizaram também uma tarefa de preenchimento de lacunas para obtenção de dados em estilo de fala semiformal (720 ocorrências). Esses dados não serão tratados neste artigo, porém o leitor poderá observá-los em RENNICKE (2015).

Os participantes foram escolhidos à base da observação dos róticos em coda. Falantes que usassem apenas fricativas posteriores em coda não foram escolhidos porque, considerando que elas constituem a pronúncia prestigiada em Minas Gerais (RENNICKE, 2011), a ocorrência de qualquer outro rótico neste contexto não era provável. Portanto, procedeu-se à gravação de falantes que utilizassem somente aproximantes ou aproximantes em combinação com fricativas posteriores. Este padrão poderia estar relacionado ou à cidade de origem do falante, ou ao tempo vivido em Lavras (que se situa na isoglossa das tendências em coda, cf. AGUILERA; SILVA, 2011). Os falantes não são de uma única origem geográfica porque a finalidade deste estudo não é explicar a variação em função da origem; pelo contrário, pretendemos propor um modelo de representação que englobe a produção e percepção de todas as variantes que coexistem em uma comunidade de fala. 


\section{Distribuição das variantes}

\section{1. $R$-forte}

Nas Tabelas 2 e 3, é possível observar que o R-forte é predominantemente glotal nos falantes analisados. Há poucas ocorrências de fricativas uvulares e cancelamento. Em posição inicial, as variantes aparecem classificadas em colunas que informam a natureza do segmento anterior (vozeado, desvozeado ou pausa) e a tonicidade do R-forte. Tanto em posição tônica como átona, é mais comum que o R-forte seja desvozeado quando precedido por um segmento desvozeado ou pausa. Conclui-se que o R-forte já não se enquadra na classe de obstruintes ou líquidas que se definem em termos de vozeamento: no caso do R-forte, o vozeamento é instável e nem sempre é assimilado pela sibilante precedente. Das 30 ocorrências de R-forte precedido por [s], o rótico foi realizado como [6] (2), [h] (26) e Ø (2); e precedido por [z] em 6 ocorrências, foi realizado como [h] (4) e [h] (2). Portanto, parece-nos que o R-forte mais vezes se assimila ao vozeamento de segmentos adjacentes (tanto vogais quanto consoantes), conforme pode ser constatado comparando as ocorrências em início de palavra, quando precedidas por segmento desvozeado ou pausa (em que predominam variantes desvozeadas), com a posição inicial precedida por segmento vozeado e a posição intervocálica (em que predominam variantes vozeadas).

TABELA 2: Variantes do R-forte em início de palavra

\begin{tabular}{|c|c|c|c|c|c|c|c|c|c|c|c|c|}
\hline \multirow{2}{*}{ Contexto } & \multicolumn{2}{|c|}{$\begin{array}{c}\text { [+voz] } \\
\text { R tônico }\end{array}$} & \multicolumn{2}{c|}{$\begin{array}{c}\text { [-voz] } \\
\text { R tônico }\end{array}$} & \multicolumn{2}{c|}{$\begin{array}{c}\text { Pausa } \\
\text { R tônico }\end{array}$} & \multicolumn{2}{c|}{$\begin{array}{c}\text { [+voz] } \\
\text { R átono }\end{array}$} & \multicolumn{2}{c|}{$\begin{array}{c}\text { [-voz] } \\
\text { R átono }\end{array}$} & \multicolumn{3}{|c|}{$\begin{array}{c}\text { Pausa } \\
\text { R átono }\end{array}$} \\
\hline Variante & $\mathrm{N}$ & $\mathbf{\%}$ & $\mathrm{N}$ & $\mathbf{\%}$ & $\mathrm{N}$ & $\mathbf{\%}$ & $\mathrm{N}$ & $\mathbf{\%}$ & $\mathrm{N}$ & $\mathbf{\%}$ & $\mathrm{N}$ & $\mathbf{\%}$ \\
\hline $\mathrm{h}$ & 68 & $\mathbf{8 6 , 1}$ & 1 & $\mathbf{7 , 7}$ & 1 & $\mathbf{2 0 , 0}$ & 188 & $\mathbf{9 4 , 9}$ & 13 & $\mathbf{3 1 , 0}$ & 11 & $\mathbf{3 5 , 5}$ \\
\hline $\mathrm{h}$ & 7 & $\mathbf{8 , 9}$ & 12 & $\mathbf{9 2 , 3}$ & 2 & $\mathbf{4 0 , 0}$ & 10 & $\mathbf{5 , 1}$ & 25 & $\mathbf{5 9 , 5}$ & 20 & $\mathbf{6 4 , 5}$ \\
\hline & 2 & $\mathbf{2 , 5}$ & 0 & $\mathbf{0}$ & 0 & $\mathbf{0}$ & 0 & $\mathbf{0}$ & 0 & $\mathbf{0}$ & 0 & $\mathbf{0}$ \\
\hline$\chi$ & 2 & $\mathbf{2 , 5}$ & 0 & $\mathbf{0}$ & 2 & $\mathbf{4 0 , 0}$ & 0 & $\mathbf{0}$ & 1 & $\mathbf{2 , 4}$ & 0 & $\mathbf{0}$ \\
\hline$\varnothing$ & 0 & $\mathbf{0}$ & 0 & $\mathbf{0}$ & 0 & $\mathbf{0}$ & 0 & $\mathbf{0}$ & 3 & $\mathbf{7 , 1}$ & 0 & $\mathbf{0}$ \\
\hline Total & 79 & $\mathbf{1 0 0}$ & 13 & $\mathbf{1 0 0}$ & 5 & $\mathbf{1 0 0}$ & 198 & $\mathbf{1 0 0}$ & 42 & $\mathbf{1 0 0}$ & 31 & $\mathbf{1 0 0}$ \\
\hline
\end{tabular}


TABELA 3: Variantes do R-forte intervocálico

\begin{tabular}{|c|c|c|c|c|c|c|}
\hline Contexto & \multicolumn{2}{|c|}{ Tônico } & \multicolumn{2}{c|}{ Pretônico } & \multicolumn{2}{c|}{ Postônico } \\
\hline Variante & $\mathrm{N}$ & $\mathbf{\%}$ & $\mathrm{N}$ & $\mathbf{\%}$ & $\mathrm{N}$ & $\mathbf{\%}$ \\
\hline $\mathrm{h}$ & 37 & $\mathbf{7 8 , 7}$ & 27 & $\mathbf{8 7 , 1}$ & 26 & $\mathbf{8 1 , 3}$ \\
\hline $\mathrm{h}$ & 7 & $\mathbf{1 4 , 9}$ & 3 & $\mathbf{9 , 7}$ & 3 & $\mathbf{9 , 4}$ \\
\hline в & 0 & $\mathbf{0}$ & 0 & $\mathbf{0}$ & 0 & $\mathbf{0}$ \\
\hline$\chi$ & 2 & $\mathbf{4 , 3}$ & 0 & $\mathbf{0}$ & 0 & $\mathbf{0}$ \\
\hline$\varnothing$ & 1 & $\mathbf{2 , 1}$ & 1 & $\mathbf{3 , 2}$ & 3 & $\mathbf{9 , 4}$ \\
\hline Total & 47 & $\mathbf{1 0 0}$ & 31 & $\mathbf{1 0 0}$ & 32 & $\mathbf{1 0 0}$ \\
\hline
\end{tabular}

\section{2. $R$-fraco}

A Tabela 4 apresenta a distribuição de variantes do r-fraco intervocálico. O ponto de articulação desta categoria é alveolar, mas o vozeamento e o grau de constrição do r-fraco variam, indicando que há gradiência articulatória na sua realização. A variante mais frequente é o tepe aproximante $\left(\left[\frac{r}{\tau}\right]\right)$, uma articulação alveolar perceptivamente similar ao tepe, mas que apresenta formantes no espectrograma, indicando constrição incompleta.

TABELA 4: Variantes do r-fraco intervocálico

\begin{tabular}{|l|c|c|c|c|c|c|c|}
\hline \multicolumn{2}{|l|}{ Contexto } & \multicolumn{2}{|c|}{ Tônico } & \multicolumn{2}{c|}{ Pretônico } & \multicolumn{2}{c|}{ Postônico } \\
\hline Variante & $\mathrm{N}$ & $\mathbf{\%}$ & $\mathrm{N}$ & $\mathbf{\%}$ & $\mathrm{N}$ & $\mathbf{\%}$ \\
\hline Tepe alveolar & $\mathrm{f}$ & 231 & $\mathbf{3 7 , 7}$ & 107 & $\mathbf{4 3}$ & 298 & $\mathbf{3 2 , 3}$ \\
\hline $\begin{array}{l}\text { Tepe } \\
\text { aproximante }\end{array}$ & $\leftarrow$ & 372 & $\mathbf{6 0 , 7}$ & 133 & $\mathbf{5 3 . 4}$ & 549 & $\mathbf{5 9 . 4}$ \\
\hline $\begin{array}{l}\text { Tepe alveolar } \\
\text { desvozeado }\end{array}$ & $\mathrm{f}$ & 3 & $\mathbf{0 , 5}$ & 1 & $\mathbf{0 . 4}$ & 57 & $\mathbf{6 . 2}$ \\
\hline $\begin{array}{l}\text { Fricativa } \\
\text { alveolar }\end{array}$ & $\mathrm{I}+\mathrm{I}$ & 3 & $\mathbf{0 , 5}$ & 2 & $\mathbf{0 . 8}$ & 10 & $\mathbf{1 . 1}$ \\
\hline $\begin{array}{l}\text { Aproximante } \\
\text { alveolar }\end{array}$ & $\mathrm{I}$ & 0 & $\mathbf{0}$ & 1 & $\mathbf{0 . 4}$ & 2 & $\mathbf{0 . 2}$ \\
\hline Cancelamento & $\varnothing$ & 4 & $\mathbf{0 , 7}$ & 5 & $\mathbf{2}$ & 8 & $\mathbf{0 . 9}$ \\
\hline & Total & 613 & $\mathbf{1 0 0}$ & 249 & $\mathbf{1 0 0}$ & 924 & $\mathbf{1 0 0}$ \\
\hline
\end{tabular}


$\mathrm{Na}$ Tabela 5, aparecem as variantes do $\mathrm{r}$-fraco em encontros consonantais ( $\mathrm{Cr}$ ). Neste contexto, é frequente a inserção de um elemento vocálico entre o primeiro segmento e o r-fraco (CVr) (SILVA, CLEMENTE \& NISHIDA, 2006); de fato, ocorrências com elemento vocálico constituem $59 \%$ de todas as ocorrências de Cr. Na Tabela 5, aparecem somadas as ocorrências de cada variante do r-fraco com ou sem elemento vocálico. Da mesma forma que em contexto intervocálico, em Cr, o r-fraco é uma articulação alveolar cujo vozeamento e grau de constrição variam. Foram encontradas uma ocorrência de [r] e uma de [r] em $\mathrm{Cr}$, indicando que há, de fato, uma ligação articulatória entre [r] e [r], conforme discutido em 3.1. O cancelamento do $r$-fraco é consideravelmente mais avançado em encontros consonantais do que em contexto intervocálico, especialmente em posição postônica (em que o r-fraco se cancela em $20,4 \%$ das ocorrências, em palavras como outro e quatro).

TABELA 5: Variantes do r-fraco em encontros consonantais

\begin{tabular}{|c|c|c|c|c|c|c|c|}
\hline \multirow{2}{*}{\multicolumn{2}{|c|}{$\begin{array}{l}\text { Contexto } \\
\text { Variante }\end{array}$}} & \multicolumn{2}{|c|}{ Tônico } & \multicolumn{2}{|c|}{ Pretônico } & \multicolumn{2}{|c|}{ Postônico } \\
\hline & & $\mathrm{N}$ & $\%$ & $\mathrm{~N}$ & $\%$ & $\mathrm{~N}$ & $\%$ \\
\hline $\begin{array}{l}\text { Vibrante } \\
\text { alveolar }\end{array}$ & $r \underset{r}{r}$ & 1 & 0.1 & 0 & $\mathbf{0}$ & 1 & 0.2 \\
\hline Tepe alveolar & r Vr & 302 & 38.3 & 302 & 37.4 & 152 & 35.6 \\
\hline $\begin{array}{l}\text { Tepe } \\
\text { aproximante }\end{array}$ & 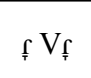 & 297 & 37.7 & 354 & 43.9 & 89 & 20.8 \\
\hline $\begin{array}{l}\text { Tepe alvolar } \\
\text { desvozeado }\end{array}$ & ${ }_{\delta} \mathrm{V}_{\delta}$ & 56 & 7.1 & 56 & 6.9 & 72 & 16.9 \\
\hline $\begin{array}{l}\text { Fricativa } \\
\text { alveolar } \\
\text { vozeada }\end{array}$ & II & 14 & 1.8 & 26 & 3.2 & 18 & 4.2 \\
\hline $\begin{array}{l}\text { Fricativa } \\
\text { alveolar } \\
\text { desvozeada }\end{array}$ & $\frac{\mathrm{I}}{\mathrm{t}}$ & 12 & 1.5 & 16 & 2 & 6 & 1.4 \\
\hline $\begin{array}{l}\text { Aproximante } \\
\text { alveolar }\end{array}$ & I & 9 & 1.1 & 11 & 1.4 & 2 & 0.5 \\
\hline Cancelamento & $\varnothing$ & 97 & 12.3 & 42 & 5.2 & 87 & 20.4 \\
\hline & Total & 788 & 100 & 807 & 100 & 427 & 100 \\
\hline
\end{tabular}




\subsection{Coda medial}

Os róticos apresentam mais variação de ponto e modo de articulação em coda silábica. As realizações variam de poucas ocorrências de tepes e fricativas alveolares para aproximantes de vários pontos de articulação, fricativas posteriores e até aproximantes aspiradas. Em coda medial, as variantes mais frequentes nos falantes analisados são as aproximantes [ $[\mathrm{I}]$, a vogal rótica [ə] e o cancelamento.

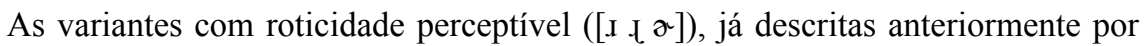
LEITE (2004), estabelecem um contínuo articulatório de lenição: $[x] \rightarrow[\tau]$ implica uma redução gestual para uma variante mais vocálica (mas não de roticidade reduzida); e $[\tau] \rightarrow[$ ə $]$ constitui uma redução temporal em que há uma sobreposição dos gestos da vogal e da aproximante em uma rima $V[x] \rightarrow[\curvearrowright]$. Esta variante ocorre mais vezes em posição pretônica e em rimas com vogal nuclear anterior (/i e $\varepsilon /)$ : o timbre da vogal nuclear é centralizado, fazendo com que os correlatos acústicos da rima inteira sejam alterados (por exemplo, perto ['pe.tu] $\rightarrow$ ['pəto] e universidade [unive.[si' dadzI] $\rightarrow$ [univərsi' dadzI] $)$.

TABELA 6: Coda medial

\begin{tabular}{|l|c|c|c|c|c|}
\hline \multicolumn{2}{|l|}{ Contexto } & \multicolumn{2}{|c|}{ Tônico } & \multicolumn{2}{c|}{ Pretônico } \\
\hline Variante & $\mathrm{N}$ & $\mathbf{0}$ & $\mathrm{N}$ & $\mathbf{\%}$ \\
\hline $\begin{array}{l}\text { Tepe alveolar } \\
\text { vozeado }\end{array}$ & $\mathrm{f}$ & 0 & $\mathbf{0}$ & 1 & $\mathbf{0 . 2}$ \\
\hline $\begin{array}{l}\text { Fricativa alveolar } \\
\text { vozeada }\end{array}$ & $\mathrm{I}$ & 0 & $\mathbf{0}$ & 2 & $\mathbf{0 . 4}$ \\
\hline $\begin{array}{l}\text { Aproximante } \\
\text { alveolar }\end{array}$ & $\mathrm{I}$ & 33 & $\mathbf{7 . 3}$ & 45 & $\mathbf{9 . 3}$ \\
\hline $\begin{array}{l}\text { Aproximante } \\
\text { palatal }\end{array}$ & $\mathrm{j}$ & 23 & $\mathbf{5 . 1}$ & 17 & $\mathbf{3 . 5}$ \\
\hline $\begin{array}{l}\text { Aproximante } \\
\text { labiovelar }\end{array}$ & $\mathrm{w}$ & 0 & $\mathbf{0}$ & 1 & $\mathbf{0 . 2}$ \\
\hline $\begin{array}{l}\text { Aproximante } \\
\text { retroflexa/arqueada }\end{array}$ & $\mathrm{I}$ & 118 & $\mathbf{2 6 . 2}$ & 76 & $\mathbf{1 5 . 7}$ \\
\hline
\end{tabular}




\begin{tabular}{|c|c|c|c|c|c|}
\hline $\begin{array}{l}\text { Vogal rótica } \\
\text { (silábica) }\end{array}$ & $a$ & 79 & 17.5 & 187 & 38.7 \\
\hline $\begin{array}{l}\text { Aproximante } \\
\text { alveolar aspirada }\end{array}$ & $\mathrm{I}^{\mathrm{h}}$ & 3 & 0.7 & 11 & 2.3 \\
\hline $\begin{array}{l}\text { Aproximante } \\
\text { palatal aspirada }\end{array}$ & $\mathrm{j}^{\mathrm{h}}$ & 1 & 0.2 & 0 & $\mathbf{0}$ \\
\hline $\begin{array}{l}\text { Aproximante } \\
\text { retroflexa/arqueada } \\
\text { aspirada }\end{array}$ & $\mathrm{l}^{\mathrm{h}}$ & 5 & 1.1 & 7 & 1.4 \\
\hline $\begin{array}{l}\text { Vogal rótica } \\
\text { aspirada }\end{array}$ & $\partial^{h}$ & 18 & 4 & 12 & 2.5 \\
\hline $\begin{array}{l}\text { Fricativa uvular } \\
\text { desvozeada }\end{array}$ & $\chi$ & 5 & 1.1 & 1 & 0.2 \\
\hline $\begin{array}{l}\text { Fricativa glotal } \\
\text { desvozeada }\end{array}$ & $\mathrm{h}$ & 19 & 4.2 & 6 & 1.2 \\
\hline $\begin{array}{l}\text { Fricativa glotal } \\
\text { vozeada }\end{array}$ & h & 20 & 4.4 & 30 & 6.2 \\
\hline Schwa & ə & 5 & 1.1 & 1 & 0.2 \\
\hline \multirow[t]{2}{*}{ Cancelamento } & $\varnothing$ & 122 & 27.1 & 86 & 17.8 \\
\hline & Total & 451 & 100 & 483 & 100 \\
\hline
\end{tabular}

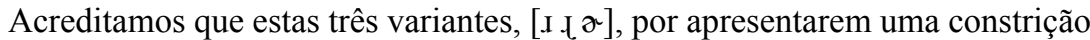
parcial e forte roticidade perceptiva, são comumente generalizadas como $r$ retroflexo/caipira nos estudos variacionistas que não se baseiam em análise acústica. Esta generalização favorece explicações das aproximantes em coda como empréstimo fonético que não se enquadra nas trajetórias de lenição em coda no PB. No entanto, um estudo que contempla a gradiência formântica das várias aproximantes ${ }^{2}$ consegue estabelecer um contínuo articulatório motivado por lenição. Além disso, há evidências de que a configuração lingual de [r] nem sempre é retroflexa: estudos sobre o inglês e o holandês (DELATTRE; FREEMAN, 1968; GICK; CAMPBELL, 2003; SCOBBIE; SEBREGTS, 2010) indicam que

$2[\mathrm{I}]$ caracteriza-se por F3 ascendente; [j] por F2 e F3 ascendentes; [.t] por F3 descendente próximo ao F2; [ə] por ser similar a [.t], porém silábico (LEITE, 2004; RENNICKE, 2015). 
esta aproximante de F3 descendente pode ser articulada com a ponta da língua retroflexa ou com o dorso da língua arqueada (e com a ponta da língua apontando para baixo), produzindo o mesmo correlato acústico. A existência de um contínuo de lenição e a variabilidade configuracional de [^] desqualificam a generalização em $r$ retroflexo e favorecem um termo mais abrangente como aproximante rótica.

As combinações de variantes, a nível individual, são variadas: 6 falantes apresentam aproximantes, vogais róticas e cancelamento, e um falante apenas aproximantes e vogais róticas. Os 7 falantes restantes também utilizam estas variantes; além delas, três deles também utilizam aproximantes aspiradas e fricativas posteriores; um utiliza aproximantes aspiradas; dois utilizam fricativas posteriores; e um falante utiliza aproximantes aspiradas, fricativas posteriores e schwa. Portanto, a convivência das duas tendências em coda - aproximantes róticas e fricativas posteriores - pode ser constatada não só nos dados gerais, mas também ao nível individual no caso de metade dos falantes analisados.

As aproximantes e vogal rótica aspiradas $\left(\left[\mathrm{I}^{\mathrm{h}} \mathrm{j}^{\mathrm{h}} \mathrm{t}^{\mathrm{h}} \boldsymbol{\gamma}^{\mathrm{h}}\right]\right)$ não têm sido descritas anteriormente como variantes no $\mathrm{PB}$, e acreditamos que a sua deteç̧ão se deve ao fato de o local de coleta de dados localizar-se na isófona. Propomos que estas variantes têm a sua origem na sobreposição de uma aspiração glotal à rima VR

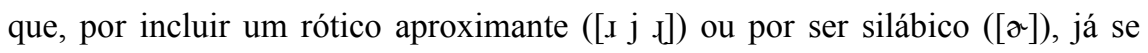
caracteriza como um ditongo ou uma vogal silábica. Em outras palavras, a rima VR já foi tão automatizada pelo falante que permite a adição de uma aspiração, provavelmente na tentativa de produzir uma pronúncia mais prestigiada. As aproximantes aspiradas não constituem uma proporção significante dos dados: elas formam $12,2 \%$ das ocorrências em coda medial; $7,7 \%$ na coda final de nominais (Tabela 7); não ocorrem na coda final de verbos; e formam 5,4\% do rótico na preposição por (Tabela 9).

\subsection{Coda final tônica}

Os resultados para coda final foram separados em nominais (substantivos e adjetivos; Tabela 7), verbos (na sua maioria infinitivos, mas incluindo também formas como quiser, for, quer; Tabela 8) e a preposição por (Tabela 9), e listados em função do segmento que segue o rótico final (consoante, vogal ou pausa). Foram omitidas deste artigo 40 ocorrências de contexto final que não entram nestas categorias: 20 da frase preposicional apesar de, 14 do pronome qualquer, e 6 paroxítonas (prefixo super-, caráter, açúcar). 
Em qualquer tipo de palavra, o cancelamento é sempre mais comum ante consoantes do que ante pausa ou vogal. A ocorrência de tepes é mais comum ante vogal, conforme a fonotática tradicional, mas é a única variante ante vogal somente em por; em nominais e verbos ocorrem aproximantes, fricativas posteriores e cancelamentos ante vogais, indicando que a lenição por fricativização e aproximantização já está avançada em nominais e verbos. O tepe foi preservado em por+V pelo fenômeno chunking, ou juntura de duas ou mais palavras para formar uma unidade fonológica (BYBEE, 2000, p. 258). A preposição é seguida de vogal em colocações fixas como por exemplo $(50,5 \%$ de todas as ocorrências pré-vocálicas) e por e-mail; por adverbiais em por aqui, por aí, por enquanto; pelo artigo indefinido em por um, por uma; por pronome pessoal em por elas; e demonstrativos em por isso, por esse, por esses, por aqueles. A ocorrência de por $+\mathrm{V}$ dá-se, portanto, em expressões fixas ou seguida de morfemas gramaticais frequentes, o que promove a juntura fonológica das palavras envolvidas, deixando o rótico de por em posição intervocálica. Chunking parece não ocorrer nos nominais, verbos, ou por $+\mathrm{C}$.

TABELA 7: Coda final em nominais

\begin{tabular}{|l|c|c|c|c|c|c|c|}
\hline \multicolumn{2}{|l|}{ Contexto } & \multicolumn{2}{|c|}{+ C } & \multicolumn{2}{c|}{+ V } & \multicolumn{2}{|c|}{+ P } \\
\hline Variante & $\mathrm{N}$ & $\mathbf{\%}$ & $\mathrm{N}$ & $\mathbf{\%}$ & $\mathrm{N}$ & $\mathbf{\%}$ \\
\hline $\begin{array}{l}\text { Tepe alveolar } \\
\text { vozeado }\end{array}$ & $\mathrm{f}$ & 0 & $\mathbf{0}$ & 10 & $\mathbf{2 3 , 3}$ & 0 & $\mathbf{0}$ \\
\hline $\begin{array}{l}\text { Tepe alveolar } \\
\text { desvozeado }\end{array}$ & $\mathrm{f}$ & 0 & $\mathbf{0}$ & 1 & $\mathbf{2 , 3}$ & 0 & $\mathbf{0}$ \\
\hline $\begin{array}{l}\text { Tepe } \\
\text { aproximante }\end{array}$ & $\mathrm{f}$ & 1 & $\mathbf{0 , 8}$ & 12 & $\mathbf{2 7 , 9}$ & 0 & $\mathbf{0}$ \\
\hline $\begin{array}{l}\text { Aproximante } \\
\text { alveolar }\end{array}$ & $\mathrm{I}$ & 5 & $\mathbf{4 , 2}$ & 0 & $\mathbf{0}$ & 0 & $\mathbf{0}$ \\
\hline $\begin{array}{l}\text { Aproximante } \\
\text { palatal }\end{array}$ & $\mathrm{j}$ & 2 & $\mathbf{1 , 7}$ & 0 & $\mathbf{0}$ & 0 & $\mathbf{0}$ \\
\hline
\end{tabular}


Representação fonológica dos róticos do Português Brasileiro: uma abordagem à base de exemplares

\begin{tabular}{|c|c|c|c|c|c|c|c|}
\hline $\begin{array}{l}\text { Aproximante } \\
\text { retroflexa/ } \\
\text { arqueada }\end{array}$ & I & 46 & 39 & 7 & 16,3 & 29 & 59,2 \\
\hline $\begin{array}{l}\text { Vogal rótica } \\
\text { (silábica) }\end{array}$ & $\alpha$ & 9 & 7,6 & 1 & 2,3 & 2 & 4,1 \\
\hline $\begin{array}{l}\text { Aproximante } \\
\text { retroflexa/ } \\
\text { arqueada } \\
\text { aspirada }\end{array}$ & $\mathrm{t}^{\mathrm{h}}$ & 1 & 0,8 & 0 & $\mathbf{0}$ & 2 & 4,1 \\
\hline $\begin{array}{l}\text { Vogal rótica } \\
\text { (silábica) } \\
\text { aspirada }\end{array}$ & $\partial^{h}$ & 1 & 0,8 & 0 & 0 & 1 & 2,0 \\
\hline $\begin{array}{l}\text { Fricativa } \\
\text { glotal } \\
\text { desvozeada }\end{array}$ & $\mathrm{h}$ & 3 & 2,5 & 1 & 2,3 & 2 & 4,1 \\
\hline $\begin{array}{l}\text { Fricativa } \\
\text { glotal vozeada }\end{array}$ & h & 9 & 7,6 & 0 & $\mathbf{0}$ & 3 & 6,1 \\
\hline Schwa & ə & 1 & 0,8 & 0 & $\mathbf{0}$ & 0 & $\mathbf{0}$ \\
\hline Cancelamento & $\varnothing$ & 40 & 33,9 & 11 & 25,6 & 10 & 20,4 \\
\hline & Total & 118 & 100 & 43 & 100 & 49 & 100 \\
\hline
\end{tabular}

TABELA 8: Coda final em verbos

\begin{tabular}{|l|c|c|c|c|c|c|c|}
\hline Contexto & \multicolumn{2}{|c|}{+ C } & \multicolumn{2}{c|}{+ V } & \multicolumn{2}{c|}{+ P } \\
\hline Variante & $\mathrm{N}$ & $\%$ & $\mathrm{~N}$ & $\%$ & $\mathrm{~N}$ & $\%$ \\
\hline $\begin{array}{l}\text { Tepe alveolar } \\
\text { vozeado }\end{array}$ & $\mathrm{r}$ & 0 & $\mathbf{0}$ & 2 & $\mathbf{0 , 5}$ & 0 & $\mathbf{0}$ \\
\hline $\begin{array}{l}\text { Tepe } \\
\text { aproximante }\end{array}$ & $\mathrm{f}$ & 0 & $\mathbf{0}$ & 9 & $\mathbf{2}$ & 0 & $\mathbf{0}$ \\
\hline
\end{tabular}




\begin{tabular}{|l|c|c|c|c|c|c|c|}
\hline $\begin{array}{l}\text { Aproximante } \\
\text { palatal }\end{array}$ & $\mathrm{j}$ & 1 & $\mathbf{0 , 2}$ & 0 & $\mathbf{0}$ & 0 & $\mathbf{0}$ \\
\hline $\begin{array}{l}\text { Aproximante } \\
\text { retroflexa/ } \\
\text { arqueada }\end{array}$ & $\mathrm{l}$ & 5 & $\mathbf{0 , 7}$ & 0 & $\mathbf{0}$ & 1 & $\mathbf{0 , 4}$ \\
\hline $\begin{array}{l}\text { Vogal rótica } \\
\text { silábica) }\end{array}$ & $\mathrm{\gamma}$ & 0 & $\mathbf{0}$ & 1 & $\mathbf{0 , 2}$ & 1 & $\mathbf{0 , 4}$ \\
\hline $\begin{array}{l}\text { Fricativa } \\
\text { glotal } \\
\text { desvozeada }\end{array}$ & $\mathrm{h}$ & 0 & $\mathbf{0}$ & 0 & $\mathbf{0}$ & 16 & $\mathbf{5 , 9}$ \\
\hline $\begin{array}{l}\text { Fricativa } \\
\text { glotal vozeada }\end{array}$ & $\mathrm{h}$ & 3 & $\mathbf{0 , 4}$ & 1 & $\mathbf{0 , 2}$ & 5 & $\mathbf{1 , 7}$ \\
\hline Schwa & ə & 3 & $\mathbf{0 , 4}$ & 0 & $\mathbf{0}$ & 1 & $\mathbf{0 , 4}$ \\
\hline Cancelamento & $\varnothing$ & 696 & $\mathbf{9 8 , 3}$ & 431 & $\mathbf{9 7 , 1}$ & 248 & $\mathbf{9 1 , 2}$ \\
\hline Total & 708 & $\mathbf{1 0 0}$ & 444 & $\mathbf{1 0 0}$ & 272 & $\mathbf{1 0 0}$ \\
\hline
\end{tabular}

TABELA 9: Coda final na preposição por

\begin{tabular}{|c|c|c|c|c|c|c|c|}
\hline \multirow{2}{*}{\multicolumn{2}{|c|}{$\begin{array}{l}\text { Contexto } \\
\text { Variante }\end{array}$}} & \multicolumn{2}{|c|}{$+\mathrm{C}$} & \multicolumn{2}{|c|}{$+\mathbf{V}$} & \multicolumn{2}{|c|}{$+\mathbf{P}$} \\
\hline & & $\mathrm{N}$ & $\%$ & $\mathrm{~N}$ & $\%$ & $\mathrm{~N}$ & $\%$ \\
\hline $\begin{array}{l}\text { Tepe alveolar } \\
\text { vozeado }\end{array}$ & I & 0 & 0 & 51 & 57,3 & 0 & 0 \\
\hline $\begin{array}{l}\text { Tepe } \\
\text { aproximante }\end{array}$ & $\underset{f}{f}$ & 0 & 0 & 38 & 42,7 & 0 & $\mathbf{0}$ \\
\hline $\begin{array}{l}\text { Aproximante } \\
\text { alveolar }\end{array}$ & $\mathrm{I}$ & 2 & 3,6 & 0 & 0 & 0 & \\
\hline $\begin{array}{l}\text { Aproximante } \\
\text { palatal }\end{array}$ & $\mathrm{j}$ & 6 & 10,9 & 0 & 0 & 0 & 0 \\
\hline $\begin{array}{l}\text { Aproximante } \\
\text { retroflexa/ } \\
\text { arqueada }\end{array}$ & $t$ & 8 & 14,5 & 0 & 0 & 1 & 33,3 \\
\hline $\begin{array}{l}\text { Vogal rótica } \\
\text { (silábica) }\end{array}$ & $a$ & 16 & 29,1 & 0 & 0 & 2 & 66,7 \\
\hline
\end{tabular}




\begin{tabular}{|l|c|c|c|c|c|c|c|}
\hline $\begin{array}{l}\text { Aproximante } \\
\text { retroflexa/ } \\
\text { arqueada } \\
\text { aspirada }\end{array}$ & $\iota^{\mathrm{h}}$ & 2 & $\mathbf{3 , 6}$ & 0 & $\mathbf{0}$ & 0 & $\mathbf{0}$ \\
\hline $\begin{array}{l}\text { Vogal rótica } \\
\text { (silábica) } \\
\text { aspirada }\end{array}$ & $\partial^{\mathrm{h}}$ & 1 & $\mathbf{1 , 8}$ & 0 & $\mathbf{0}$ & 0 & $\mathbf{0}$ \\
\hline Cancelamento & $\varnothing$ & 20 & $\mathbf{3 6 , 4}$ & 0 & $\mathbf{0}$ & 0 & $\mathbf{0}$ \\
\hline & Total & 55 & $\mathbf{1 0 0}$ & 89 & $\mathbf{1 0 0}$ & 3 & $\mathbf{1 0 0}$ \\
\hline
\end{tabular}

\section{Gradiência nos róticos}

A seção 5 mostrou que os róticos do $\mathrm{PB}$ são um grupo de sons heterogêneo: os contextos de R-forte englobam fricativas posteriores; r-fraco (intervocálico e em encontros consonantais) é principalmente uma articulação apical; e em coda podem ocorrer variantes apicais, posteriores, aproximantes (que podem ainda ser aspiradas), e também vogais róticas como resultado da sobreposição de gestos em rimas VR. A Tabela 10 resume as mudanças diacrônicas e a variação sincrônica nos róticos, especificando os seguintes aspectos: a coluna "Tipo" refere-se ao ponto de articulação ou direção da lenição no espaço articulatório; "Mudança" especifica cada passo de mudança sonora; "Motivação" indica se a mudança tem motivação perceptiva ou de automatização gestual (ou as duas); e "Redução" define se a lenição ocorreu por redução gestual ou temporal.

TABELA 10: Mudanças sonoras nos róticos do PB

\begin{tabular}{|c|c|c|c|c|c|}
\hline \multirow{2}{*}{ Tipo } & \multirow[b]{2}{*}{ Mudança } & \multicolumn{2}{|c|}{ Motivação } & \multicolumn{2}{|c|}{ Redução } \\
\hline & & Percepção & $\begin{array}{c}\text { Automatização } \\
\text { pessoal }\end{array}$ & Gestual & Temporal \\
\hline \multirow{4}{*}{$\begin{array}{l}\text { Lenição do } \\
\text { gesto apical }\end{array}$} & $r \rightarrow r$ & $\mathrm{X}$ & $\mathrm{X}$ & & $\mathrm{X}$ \\
\hline & $f \rightarrow f r$ & & $\mathrm{X}$ & $\mathrm{X}$ & \\
\hline & $\underset{f}{\int} \rightarrow \underset{t}{I} \mathrm{I}$ & & $\mathrm{X}$ & $\mathrm{X}$ & \\
\hline & $\mathrm{I} I \rightarrow \mathrm{I}$ & & $\mathrm{X}$ & $\mathrm{X}$ & \\
\hline
\end{tabular}




\begin{tabular}{|c|c|c|c|c|c|}
\hline \multirow{4}{*}{$\begin{array}{l}\text { Vocalização ou } \\
\text { centralização }\end{array}$} & $\mathrm{I} \rightarrow \mathrm{j}$ & & $\bar{X}$ & $\mathrm{X}$ & \\
\hline & $\mathrm{I} \rightarrow \mathrm{t}$ & & $\mathrm{X}$ & $\mathrm{X}$ & \\
\hline & $\mathrm{t} \rightarrow \partial \mathrm{w}$ & & $X$ & $X$ & \\
\hline & $t \rightarrow a$ & & $\mathrm{X}$ & & $\mathrm{X}$ \\
\hline Posteriorização & $r \rightarrow R$ & $\mathrm{X}$ & & & \\
\hline \multirow{2}{*}{$\begin{array}{l}\text { Lenição do } \\
\text { gesto dorsal }\end{array}$} & $\mathrm{R} \rightarrow \mathrm{B} \chi$ & & $X$ & $\mathrm{X}$ & \\
\hline & в $\chi \rightarrow h \mathrm{~h}$ & & $\mathrm{X}$ & $\mathrm{X}$ & \\
\hline $\begin{array}{l}\text { Sobreposição } \\
\text { de variantes }\end{array}$ & $\begin{array}{l}\text { Aproximante } \\
\rightarrow \\
\text { aproximante } \\
\text { aspirada }\end{array}$ & & $\mathrm{X}$ & & \\
\hline $\begin{array}{l}\text { Lenição de } \\
\text { magnitude }\end{array}$ & $\begin{array}{c}\text { Qualquer } \\
\text { variante } \rightarrow \varnothing\end{array}$ & & $\mathrm{X}$ & $\mathrm{X}$ & \\
\hline
\end{tabular}

$\mathrm{Na}$ seção 2.2., sugerimos que a causa da lenição seja a automatização neuromotora de gestos articulatórios por meio da repetição. A automatização é a força por trás da maior parte das mudanças na Tabela 10, porém há duas mudanças que julgamos terem uma motivação perceptiva. Conforme discutido na seção 3.1., $[\mathrm{r}] \rightarrow[\mathrm{r}]$ envolve uma redução temporal de múltiplas batidas para uma só, mas esta redução não tem relação articulatória direta por causa da diferença configuracional essencial entre a vibrante e o tepe. Postulamos que uma vibrante de uma batida sirva como articulação intermediária que, por semelhança perceptiva, é reinterpretada como um tepe. A segunda mudança de motivação perceptiva é a posteriorização $[\mathrm{r}] \rightarrow[\mathrm{R}]$; na seção 3.2. explicitamos que a permuta do ponto de articulação não resulta em um segmento articulatoriamente mais reduzido.

$\mathrm{Na}$ Tabela 10, as mudanças motivadas por automatização gestual envolvem quase sempre o mecanismo de redução gestual: a passagem de uma articulação de constrição completa para uma constrição incompleta ou a perda do vozeamento, como constatamos na trajetória do tepe para o tepe aproximante ou tepe desvozeado, passando para uma articulação fricativa, e por último, aproximante. Da mesma forma, a vibrante uvular (não encontrada no corpus deste estudo) deu lugar a fricativas uvulares, que por sua vez se enfraqueceram para fricativas glotais por debucalização. $\mathrm{O}$ cancelamento de qualquer rótico também pode ser considerado uma redução gestual. A diversificação de $[\mathrm{I}]$ para outras aproximantes e schwa ([j] l $\mathrm{W}$ ə]), em coda, reflete a gradiência entre as realizações aproximantes: pequenas modificações nas transições formânticas de núcleo para coda mantêm um ditongo 
no nível fonético e não prejudicam a percepção: de acordo com as premissas da Fonologia de Exemplares, a percepção ocorre ao nível da palavra (e não de sons), possibilitando discrepâncias e mudanças significantes no nível segmental. [-] $\rightarrow$ [ð] apresenta uma redução temporal, sendo que resulta da antecipação do gesto (retroflexo ou arqueado) durante a vogal nuclear, produzindo um F3 baixo durante toda a duração da rima.

Propomos que o surgimento de aproximantes aspiradas na variedade analisada não represente um caso de lenição. Embora resultem de automatizações prévias — por um lado, da aproximantização e as vogais róticas como consequência, e por outro lado, das fricativas posteriores debucalizadas - não parece plausível que a "combinação" de duas tendências represente uma lenição. A explicação que encontramos para este fenômeno é o caráter vocálico de rimas VR, no final das quais o falante pode adicionar uma leve aspiração com o objetivo de alcançar uma pronúncia mais prestigiada.

Este resumo das motivações e mecanismos das mudanças no sistema dos róticos deixa claro que se trata de uma classe de sons gradiente e fragmentada em termos de ponto e modo de articulação e contexto fonológico, e as causas dessa fragmentação são complexas, envolvendo fatores perceptivos e de redução articulatória. Os róticos do PB já não se definem em termos de traços compartilhados; a definição dos róticos como classe será abordada seção 7 .

\section{Róticos como classe em um modelo de exemplares}

Análises de cunho formalista (vide seção 3.4.) defendem uma representação subjacente única - quer /r/, quer / $/$ / — da qual a variantes posicionais dos róticos se derivam através de um sistema complexo de regras. No entanto, é questionável basear uma definição de classe em uma variante praticamente inexistente na variedade analisada (duas ocorrências de [r] [r] ) ou em geminadas subjacentes em posição intervocálica (/r.r/ ou /r.r/) que nunca tomam forma na superfície. Apoiamo-nos em SEBREGTS (2014,p. 234-235) para enfatizar que o falante leigo não tem conhecimento das mudanças diacrônicas que levaram à distribuição sincrônica de variantes, nem necessariamente manifesta todas as formas intermediárias que possibilitariam a descoberta de traços em comum. Diante da variação fonética apresentada neste artigo, seria uma tarefa fútil procurar definir traços ou outras características em comum para todos os membros da classe, já que essas características deixaram de existir e o usuário da língua não tem acesso a elas. 
Dentro dos moldes da Fonologia de Exemplares, sugerimos que a representação fonológica dos róticos no PB tenha uma origem lexical. A forma fonética de uma palavra é armazenada junto com o seu significado, o que cria conexões fonéticas, morfológicas e semânticas com outras palavras da língua que tenham semelhanças fonotáticas ou de significado. A partir dessas conexões, o falante forma generalizações probabilísticas sobre variantes posicionais. Isto é, o acesso à forma fonética das palavras é imediata: é inerente às palavras, e não é gerada por regras a partir de uma forma subjacente.

A Figura 1 ilustra, de forma simplificada, a possível rede de variantes róticas na mente de um falante, abstraída das representações lexicais. Variantes mais frequentes, que teriam a representação mais forte, aparecem em caracteres maiores, enquanto variantes mais periféricas aparecem em caracteres menores, simbolizando o seu peso estatístico. É possível observar que as variantes de R-forte e r-fraco podem ocorrer em coda, além de outras variantes que são específicas deste último contexto. Nesta variedade do PB, as variantes de R-forte e r-fraco não se sobrepõem e são foneticamente muito distantes. Visto que a coda é o único contexto unificador em que todas as variantes podem ocorrer, propomos que existe um "quase-contraste" (LADD, 2006; SCOBBIE; STUART-SMITH, 2008) entre $\mathrm{R}$-forte e r-fraco. Um quase-contraste significa que duas categorias fonológicas podem contrastar em alguns (mas não todos os) contextos; que esse contraste depende fortemente de idioleto, socioleto e comunidade de fala; e que falantes podem ter soluções alternativas e intermediárias para categorias ambíguas sem limites nítidos.

FIGURA 1: Representação fonológica dos róticos no PB

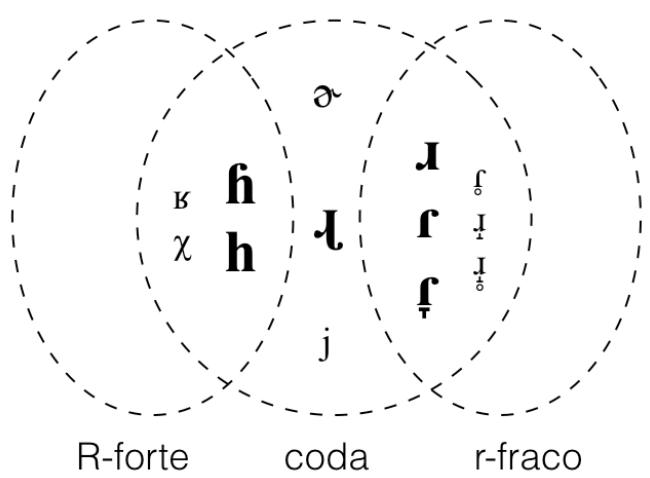


Propomos que a classe dos róticos no PB seja definida não como classe natural, mas como classe à base de relações de parentesco (family relationships) — ideia já aplicada ao holandês por SEBREGTS (2014). Diferentemente da noção de semelhança de parentesco (family resemblance) em que cada membro da classe compartilha algum traço com outro membro (A com B, B com C, C com D, etc.), sugerida por LINDAU (1985) como fundamento da classe dos róticos, em uma rede de relações de parentesco podem existir variantes cuja ligação de semelhança com outras variantes tenha se tornado obscura. Por exemplo, somente os membros A e D de uma classe ocorrem na língua, e os passos de semelhança em cadeia, $\mathrm{A} \rightarrow \mathrm{B}, \mathrm{B} \rightarrow \mathrm{C}$ e $\mathrm{C} \rightarrow \mathrm{D}$, podem não ser acessíveis ao falante sincrônico. Enfatizamos que a noção de relações de parentesco deve ser completada com a informação de que essas relações são sempre específicas de uma língua. As trajetórias de mudança nos róticos ocorrem sempre dentro do sistema fonológico de uma língua, fazendo com que o sistema rótico de cada língua se desenvolva de maneira única. Os róticos do português e do espanhol - duas línguas com um quase-contraste muito semelhante entre R-forte e r-fraco -têm hoje em dia classes róticas muito diferentes. O português desenvolveu um vasto repertório de róticos, enquanto no espanhol, os róticos mantêm-se principalmente apicais ([r $\mathrm{r}]$ ), e as fricativas posteriores ([в $\chi$ h h] etc., que são róticas no $\mathrm{PB}$ ) são variantes da categoria nãorótica $/ \mathrm{x} /$. Portanto, a classe dos róticos estabelece-se de maneira dinâmica dentro do sistema de oposições fonológicas de cada língua.

\section{Conclusões}

Este artigo demonstrou, por meio da observação de dados acústicos, que a variedade do PB analisada conta com pelo menos 21 variantes fonéticas para a classe dos róticos. Esta quantidade de variantes de diferentes pontos e modos de articulação destaca-se entre as línguas do mundo e assemelha o repertório de línguas como o holandês (SEBREGTS, 2014), conhecido pela sua variação rótica.

A lenição (ou redução) articulatória, que ocorre por diminuição do gesto articulatório ou por realinhamento temporal, é a principal força por trás da mudança diacrônica (que levou ao atual contraste intervocálico de fricativa posterior vs. tepe anterior) e da variação sincrônica. A análise acústica facilita o estabelecimento de trajetórias de lenição: como exemplo, mencionemos a mudança do tepe para aproximantes e vogal rótica $([\mathrm{r}] \rightarrow[\mathrm{f}] \rightarrow[\mathrm{I}] \rightarrow[\mathrm{I}] \rightarrow[\mathrm{x}] \rightarrow[\mathrm{\gamma}]$ ), que torna 
evidente o caráter gradiente da mudança sonora e das representações fonológicas. Consideramos que a observação da gradiência é a principal contribuição do presente estudo, pois argumenta contra a noção de que a mudança sonora se dá por meio de regras aplicadas à forma subjacente. A mudança sonora nunca pode ser cancelada já que ela modifica de maneira permanente as representações lexicais. A mudança sonora e as alterações do sistema fonológico acontecem de forma gradual, motivadas na maioria das vezes pela lenição. A única mudança no sistema dos róticos, sem aparente motivação por redução, é $[\mathrm{r}] \rightarrow[\mathrm{R}]$. Esse impulso inicial para a posteriorização e suas motivações merecem ser estudados mais detalhadamente.

Não há um traço fonético comum a todos os membros da classe dos róticos; por este motivo, a classe dos róticos baseia-se em relações de parentesco (e não de semelhança). Essas relações formam uma rede de variantes com ligações históricas, mas nem todas essas ligações são acessíveis ao falante leigo. A sobreposição de variantes em coda, ao mesmo tempo em que elas se contrastam em outros contextos, faz com que o status fonológico atual do R-forte e r-fraco categorias sem limites nítidos - seja um status de quase-contraste.

\title{
The phonological representation of rhotics in Brazilian Portuguese: an exemplar-based approach.
}

\begin{abstract}
This paper examines the variation of rhotics, or r-sounds, in a corpus of Brazilian Portuguese collected in Lavras (Minas Gerais). Acoustic analysis revealed 21 variants, which involve trills, taps, fricatives, approximants and aspirated approximants in alveolar, palatal, retroflex/bunched, uvular and glottal places of articulation. The phonetic gradience observed in all r-contexts is motivated by lenition, which can take the form of gestural reduction or retiming. According to Exemplar-Based Phonology, lenition occurs as the result of the automation of neutomotor patterns. Based on the gradience observed in the data, supplemented by information on historical sound changes in rhotics, we argue that the rhotic class can be justified not as a natural class, but as a network of language-specific family relations. The connections between different rhotics are not always present in the speech of individuals, but they can be sought in such a network since the synchronic variation makes it impossible to define this class in terms of shared features. More importantly, this network is language-specific because it has been formed in interaction with other elements in the phonological
\end{abstract}


system. The overlap of front and back rhotics in syllable codas suggest that there is a "quasi-contrast" between strong-R and weak-r in Portuguese.

Keywords: Rhotics. Exemplar-based phonology. Lenition. Phonological class.

\section{Referências}

ABAURRE, M. B. M.; SANDALO, M. F. S. Os róticos revisitados. In: HORA, D.; COLLISCHON, G. (Eds.) Teoria linguística: Fonologia e outros temas. Editora Universitária/UFPB, 2003. p. 144-180.

AGUILERA, V. A.; SILVA, H. C. "Dois momentos do /r/ retroflexo em Lavras - MG: no Atlas Linguístico de Minas Gerais e nos dados do projeto do Atlas Linguístico do Brasil”. Diadorim, v. 8, p. 125-142, 2011.

ALMEIDA, M. M. S. “As consoantes do português falado no Vale do Cuiabá”. Signum - Estudos da Linguagem, v. 7, p. 149-163, 2004.

BARRY, W. "Another R-tickle". Journal of the International Phonetic Association, v. 27, p. 35-45, 1997.

BAUER, L. "Lenition revisited". Journal of Linguistics, v. 44, p. 605-624, 2008.

BLECUA FALGUERAS, B. Las vibrantes del español: manifestaciones acústicas y procesos fonéticos. Tese (Doutorado) - Universitat Autònoma de Barcelona, 2001.

BOERSMA, P.; WEENINK, D. Praat: doing phonetics by computer. Programa de computador, versão 6.0.15. Disponível em < wWw.praat.org $>, 2016$.

BONET, E.; MASCARÓ, J. On the representation of contrasting rhotics. In: MARTÍNEZ-GIL, F.; MORALES-FRONT, A. (Eds.) Issues in the Phonology and Morphology of the Major Iberian Languages. Washington, D.C.: Georgetown University Press, 1997. p. 103-126.

BROWMAN, C. P.; GOLDSTEIN, L. "Articulatory phonology: An overview". Phonetica, v. 49, p. 155-180, 1992.

BROWMAN, C. P.; GOLDSTEIN, L. "Towards an articulatory phonology". Phonology Yearbook, v. 3, p. 219-252, 1986.

BYBEE, J. Lexicalization of sound change and alternating environments. In: BROE, M.; PIERREHUMBERT, J. P. (Eds.) Laboratory phonology V: 
Acquisition and the lexicon. Cambridge: Cambridge University Press, 2000. p. 250-268.

BYBEE, J. Phonology and language use. Cambridge University Press, 2001.

BYBEE, J. "From usage to grammar: the mind's response to repetition". Language, v. 82, n. 4, p. 711-733, 2006.

BYBEE, J. Language, usage and cognition. Cambridge University Press, 2010. CALLOU, D. M. I.; LEITE, Y.; MORAES, J. Processo(s) de enfraquecimento consonantal no português do Brasil. In: ABAURRE, M. B. M.; RODRIGUES, A. C. S. (Eds.) Gramática do português falado: Novos estudos descritivos, vol.8. Campinas: Unicamp, 2002.p. 537-555.

CALLOU, D. M. I.; MORAES, J.; LEITE, Y. Variação e diferenciação dialetal: a pronúncia do /r/ no português do Brasil. In: KOCH, I. (Ed.) Gramática do português falado, v. 6. Campinas: Unicamp, 1996. p. 465-493.

CATFORD, J. Fundamental problems in phonetics. Edinburgh University Press, 1977.

CRISTÓFARO-SILVA, T. Modelos multirrepresentacionais em fonologia. In: MARCHEZAN, R. C.; CORTINA, R. (Org.). Os fatos da linguagem, esse conjunto heteróclito. 1.ed. Araraquara: Cultura Acadêmica - FCL-UNESP Laboratório Editorial, 2006. p. 171-186.

DELATTRE, P.; FREEMAN, D. C. “A dialect study of American r's by X-ray motion picture”. Linguistics, v. 44, p. 29-68, 1968.

GICK, B.; CAMPBELL, F. Intergestural timing in English /r/. In: Proceedings of the XVth International Congress of Phonetic Sciences. Barcelona: Universitat Autònoma de Barcelona, 2003. p. 1911-1914.

GONÇALVES VIANA, A. R. Portugais: phonétique et phonologie, morphologie, textes. Leipzig: Teubner, 1903.

GONÇALVES VIANA, A. R. Estudos de fonética portuguesa. Lisboa: Imprensa Nacional-Casa da Moeda, 1973.

HUBACK, A. P. S. "Cancelamento do (r) final em nominais: uma abordagem difusionista". Scripta, v. 9, n. 18, p. 11-28, 2006.

JOHNSON, K. Speech perception without speaker normalization: An exemplar model. In: JOHNSON, K.; MULLENNIX, J. (Eds.) Talker Variability in Speech Processing. San Diego: Academic Press, 1997. p. 145-165. 
JOHNSON, K. Decisions and mechanisms in exemplar-based phonology. In: SOLE, M.; BEDDOR, P.; OHALA, M. (Eds.) Experimental Approaches to Phonology. Oxford University Press, 2007. p. 25-40.

LADD, D. R. "Distinctive phones" in surface representation. In: GOLDSTEIN, L.; WHALEN, D.; BEST, C. (Eds.) Laboratory Phonology 8. Berlin: de Gruyter, 2006. p. 3-26.

LASS, R. Phonology. An introduction to basic concepts. Cambridge University Press, 1984.

LEITE, C. M. Atitudes linguïsticas: a variante retroflexa em foco. Dissertação (Mestrado) - Instituto de Estudos da Linguagem, Universidade Estadual de Campinas, Campinas, 2004.

LEITE, C. M. O /R/ em posição de coda silábica no falar campineiro. Tese (Doutorado) - Instituto de Estudos da Linguagem, Universidade Estadual de Campinas, Campinas, 2010.

LIMA, M. M. de O. As consoantes róticas no Português Brasileiro com notas sobre as róticas das variedades de Goiânia, Goiatuba e Uberlândia. Dissertação (Mestrado) - Instituto de Letras, Departamento de Linguística, Português e Línguas Clássicas, Universidade de Brasília, 2013.

LINDAU, M. The story of /r/. In: LADEFOGED, P.; FROMKIN, V. (Eds.) Phonetic linguistics: essays in honor of Peter Ladefoged. Orlando: Academic Press, 1985. p. 157-168.

MATEUS, M. H. M.; D’ANDRADE, E. The Phonology of Portuguese. Oxford University Press, 2000.

MATEUS, M. H. M.; RODRIGUES, C. A vibrante em coda no português. In: HORA, D.; COLLISCHONN, D. (Org.). Teoria Linguística: Fonologia e Outros Temas. João Pessoa: Universidade Federal da Paraíba, 2003. p. 181-199.

MATTOSO CAMARA JR., J. Para o estudo da fonêmica portuguesa. Rio de Janeiro: Organização Simões, 1953.

MONARETTO, V. N. O.; QUEDNAU, L. R.; HORA, D. As consoantes do português. In: BISOL, L. (Ed.) Introdução a estudos de fonologia do português brasileiro. Porto Alegre: EDIPUCRS, 2005. p. 207-242.

MOWREY, R.; PAGLIUCA, W. "The reductive character of articulatory evolution”. Rivista di Linguistica, v. 7, n. 1, p. 37-124, 1995.

NOLL, V. O português brasileiro: formação e contrastes. São Paulo: Globo, 2008. 
NOLL, V. Mudanças na realização de / $/ \bar{r} /, / r /$ em português. In: LOBO, T. C. F.; CARNEIRO, Z.; SOLEDADE, J.; ALMEIDA, A.; RIBEIRO, S. (Eds.) ROSAE: linguística histórica, história das línguas e outras histórias. Salvador: EDUFBA, 2012. p. 337-348.

OLIVEIRA, M. A. Phonological variation and change in Brazilian Portuguese: the case of the liquids. Tese (Doutorado) - University of Pennsylvania, Philadelphia, 1983.

OLIVEIRA, M. A. "Reanalisando o processo de cancelamento do (r) em final de sílaba”. Revista de Estudos Linguísticos, v. 6, n. 2, p. 31-58, 1997.

OUSHIRO, L.; MENDES, R. B. "A pronúncia de (-r) em coda silábica no português paulistano". Revista do GEL, v. 8, n. 2, p. 66-95, 2013.

PIERREHUMBERT, J. Exemplar dynamics: Word frequency, lenition, and contrast. In: BYBEE, J.; HOPPER, P. (Eds.) Frequency effects and the emergence of linguistic structure. Amsterdã e Filadélfia: John Benjamins, 2001. p. 137-157. PIERREHUMBERT, J. Word-specific phonetics. In: GUSSENHOVEN, C.; WARNER, N. (Eds.) Laboratory Phonology 7. Berlim e Nova York: Mouton de Gruyter, 2002. p. 101-139.

RECASENS, D. "Weakening and strengthening in Romance revisited". Rivista di Linguistica, v. 14, n. 2, p. 327-373, 2002.

RENNICKE, I. "The retroflex $r$ of Brazilian Portuguese: theories of origin and a case study of language attitudes in Minas Gerais". Linguística: Revista de Estudos Linguísticos da Universidade do Porto, v. 6, n. 1, p. 149-170, 2011.

RENNICKE, I. Variation and Change in the Rhotics of Brazilian Portuguese. Tese (Doutorado) . Faculdade de Letras, Universidade Federal de Minas Gerais e Departamento de Línguas Modernas, Universidade de Helsinque, 2015.

RENNICKE, I.; MARTINS, P. T. As realizações fonéticas de /R/ em português europeu: análise de um corpus dialectal e implicações no sistema fonológico. In: SILVA, F.; FALÉ, I.; PEREIRA, I. (Org.) Textos Seleccionados do XXVI Encontro da Associação Portuguesa de Linguística. Coimbra: APL, 2013. p. 509-523.

RIBEIRO, C. M. da R. (2011) “O comportamento geolinguístico do (r) posvocálico nos atlas brasileiros publicados". Letras Escreve - Revista de Estudos Linguísticos, v. 1, n. 1, p. 6-21, janeiro-junho, 2011.

SCOBBIE, J. M.; SEBREGTS, K. Acoustic, articulatory, and phonological 
perspectives on allophonic variation of /r/ in Dutch. In: FOLLI, R.; ULBRICH, C. (Eds.) Interfaces in Linguistics: New Research Perspectives. Oxford University Press, 2010. p. 257-277.

SCOBBIE, J. M.; STUART-SMITH, J. Quasi-phonemic contrast and the fuzzy inventory: examples from Scottish English. In: AVERY, P.; DRESHER, B. E.; RICE, K. (Eds.) Contrast in Phonology: Theory, Perception, Acquisition, Phonology and phonetics (13). Berlim: Mouton de Gruyter, 2008. p. 87-113.

SEBREGTS, K. The sociophonetics and phonology of Dutch $\mathbf{r}$. Tese (Doutorado) - Department of Languages, Literature and Communication, Utrecht University, 2014.

SILVA, A. H. P.; CLEMENTE, F. C.; NISHIDA, G. "Para a representação dinâmica do tap em grupos e codas: evidências acústicas". Revista Virtual de Estudos da Linguagem - ReVEL, v. 4, n. 7, 2006.

STURTEVANT, E. H. The pronunciation of Greek and Latin. The sounds and accents. Chicago: The University of Chicago Press, 1920.

ZÁGARI, M. R. Os falares mineiros: esboço de um Atlas Linguístico de Minas Gerais. In: AGUILERA, V. (Ed.) A geolinguística no Brasil: caminhos e perspectivas. Londrina: Ed. UEL, 1998. p. 31-54.

Submetido: $30 / 03 / 2016$

aceite: $13 / 07 / 2016$ 